\title{
Vom Ursprung des Desaultverbandes
}

\author{
Von Markwart Michler, Bonn
}

Desault, den man als Schrittmacher der modernen klinischen Chirurgie in Paris und als Vater einer ganzen Genealogie französischer Chirurgen bezeichnen kann, hat sich auch um die Lehre von den Frakturen und Luxationen Verdienste erworben ${ }^{1}$. Neben bestimmten Bruchformen der Extremitäten widmete er den Frakturen des Schultergürtels seine besondere Aufmerksamkeit, und diesen Bemühungen verdanken wir die Erfindung des nach ihm benannten Bindenverbandes zur Ruhigstellung eines Schlüsselbeinbruches. Die Ergebnisse seiner Forschungen, zunächst in dem von ihm begründeten Journal de Chirurgie verstreut und teilweise durch seine Mitarbeiter veröffentlicht ${ }^{2}$, wurden posthum von seinem ihm in verehrender Liebe ergebenen Schüler Bichat sachlich geordnet und durch Vorlesungsnotizen ergänzt als Euvres chirurgicales de P.J. Desault herausgegeben. Hier findet sich auch eine genaue Beschreibung des «Desault» mit anschaulichen Bildtafeln (Abb. 1) ${ }^{3}$. Aber der Verband, heute fast nur noch bei traumatischen Schulterluxationen in Gebrauch ${ }^{4}$, blieb in seinem ursprünglichen Zweck, eine Claviculafraktur in reponierter Stellung zu fixieren, auch zu Desaults Zeiten nicht unumstritten ${ }^{5}$, war doch die Ruhigstellung dieser

${ }^{1}$ Vgl. W. v. Brunn, Kurze Geschichte der Chirurgie. Berlin 1928, S. 239f. - I. DE Fourmestraux, Histoire de la Chirurgie Française (1790-1920). Paris 1934, S. 14. - J. RoGHARD, Histoire de la Chirurgie Française au XIX $X^{e}$ siècle. Paris 1875, S. $5 \mathrm{f}$.

${ }^{2}$ Journal de Chirurgie par M. Desault, Chirurgien en Chef de l'Hôtel-Dieu de Paris. Paris 1791, Tome premier. Kasuistische Berichte über die Claviculafraktur von Garnier. (S. $136 \mathrm{ff.})$ mit Beschreibung der Verbandsmethode (S. 139f.), die Desault bereits um 1765 erfunden und bekanntgemacht hatte.

${ }^{3}$ Euvres chirurgicales de P.J. Desault, Ouvrage publié par Xav. Bichat, son élève. Paris 1798, Tom. I, S. 71f. und S. $82 \mathrm{f}$. mit der wiedergegebenen Bildtafel.

${ }^{4}$ Der «Desault» als Fixation nach Schulterluxation: Vgl. K. Daubenspeck, Die Luxation der Schulter, in: G. Hohmann, M. Hackenbroch, K. Lindemann, Handbuch der Orthopädie. Stuttgart 1959, Bd. III, S. 285 f., der die Anwendung dieses Verbandes allerdings auf Deutschland einschränkt; ferner G. Schumanv, Frakturen und Verrenkungen im Bereich der oberen Gliedmaße, in: P. F. Matzen, Lehrbuch der Orthopädie. Berlin 1959, Bd. II, S. 222. Gegen eine Ruhigstellung der Claviculafraktur im Desault: Derselbe ebenda S. 186.

${ }^{5}$ Vgl. die deutsche Ausgabe von Bichats Buch: J. P. Desaults Chirurgischer Nachlaß, übersetzt von G. WARDEnBURg. Göttingen 1800, S. $122 \mathrm{ff}$. - Dabei dürfte es von allge- 
Verletzung ein Problem, um dessen Schwierigkeiten bereits die hippokratischen Ärzte wußten ${ }^{6}$ und das von der antiken Medizin ungelöst an die folgenden Jahrhunderte weitergegeben wurde. Denn die Hippokratiker und nach ihnen das gesamte Altertum bedienten sich vorwiegend der Schulter-Spica und von ihr abgeleiteter Verbände, kombinierten sie mit zusätzlichen Thoraxbandagen ${ }^{7}$ und gaben diese Verfahren auf dem Umweg über die arabische Heilkunde an unsere mittelalterliche Medizin weiter. Ihre Unzulänglichkeit spiegelt sich am klarsten in den stets von neuem unternommenen Modifikationsversuchen wieder, denen sie bei so hervorragenden Chirurgen wie etwa Guy de Chauliac oder Ambroise Paré unterworfen waren; Heister schließlich nahm seine Zuflucht zu einer T-förmigen Rückenschiene aus Eisen ${ }^{8}$. Grundsätzlich aber blieben die Abkömmlinge der Spica bis zu Desault in Gebrauch ${ }^{9}$, und so dürfte dessen Verband seinerzeit auch für die Claviculafraktur einen Fortschritt bedeutet haben; denn wenn er auch keine vollkommene Lösung dieser Frage brachte, wie Wardenburg noch meinte ${ }^{10}$, so hat seine Anwendung doch zweifelsohne zu neuen Erkenntnissen geführt.

meinerem Interesse sein, daß W ARDENBURG das Werk nicht nur in die deutsche Sprache übertrug, sondern einem jeden Abschnitt zusätzliche eigene Kapitel beifügte, in denen Desaults Lehren kritisch beleuchtet und zur damaligen deutschen Chirurgie in Beziehung gesetzt wurden. Wardenburg selbst war als Freund Bichats zwar ein Anhänger von DeSaults Lehren, aber es kommen auf diese Weise doch die wichtigsten sachlichen Gegenstimmen zu Wort; ein Verfahren, das der weiteren Entwicklung der Chirurgie sicherlich sehr förderlich war.

${ }^{6}$ De articulis c. 14. (Littré IV $120 \mathrm{f}$.).

${ }^{7}$ De articulis, loc. cit.; Celsus, De re medica 1. VIII c. 8, CML I, p. 386f.; Galen, De fasciis c. 77 (XVIII A 813 ED. KüHN); hinsichtlich des Verbandes weniger klar: Paulus von Aegina 1. VI c. 93, CMG IX 2.

${ }^{8}$ Auf Ambroise Paré wird im folgenden noch zurückgekommen (Ambrosius Paraeus, Opera Chirurgica. Frankfurt 1594, 1. XIV c. 8: De fractura iuguli, p. 407 [in der genannten Edition steht versehentlich auf der angegebenen Seite als Nummer des Buches: XIII]). Heisters T-Schiene siehe: D. L. Heister, Institutiones Chirurgicae. Amsterdam 1750, Tab. VIII (p. 210).

${ }^{9}$ Chirurgische Lehrbücher, die nur ein reichliches halbes Jahrhundert vor Desaults Erfindung veröffentlicht sind, geben noch immer einen Spica-Abkömmling, die sogenannte capeline zur Fixation an; dieser Verband besteht aus Schulter-Spica-Touren und Zirkulärtouren um den Thorax. Vgl. z.B.: J. B. VERDÜcK, Chirurgische Schriften. Leipzig 1712, Teil I, S.418, oder: Bourdelot, Traité complet des opérations de chirurgie. Paris 1695 , S. 710 .

${ }^{10}$ G. WARden burg, Desaults Chirurgischer Nachlaß, S. 127. 


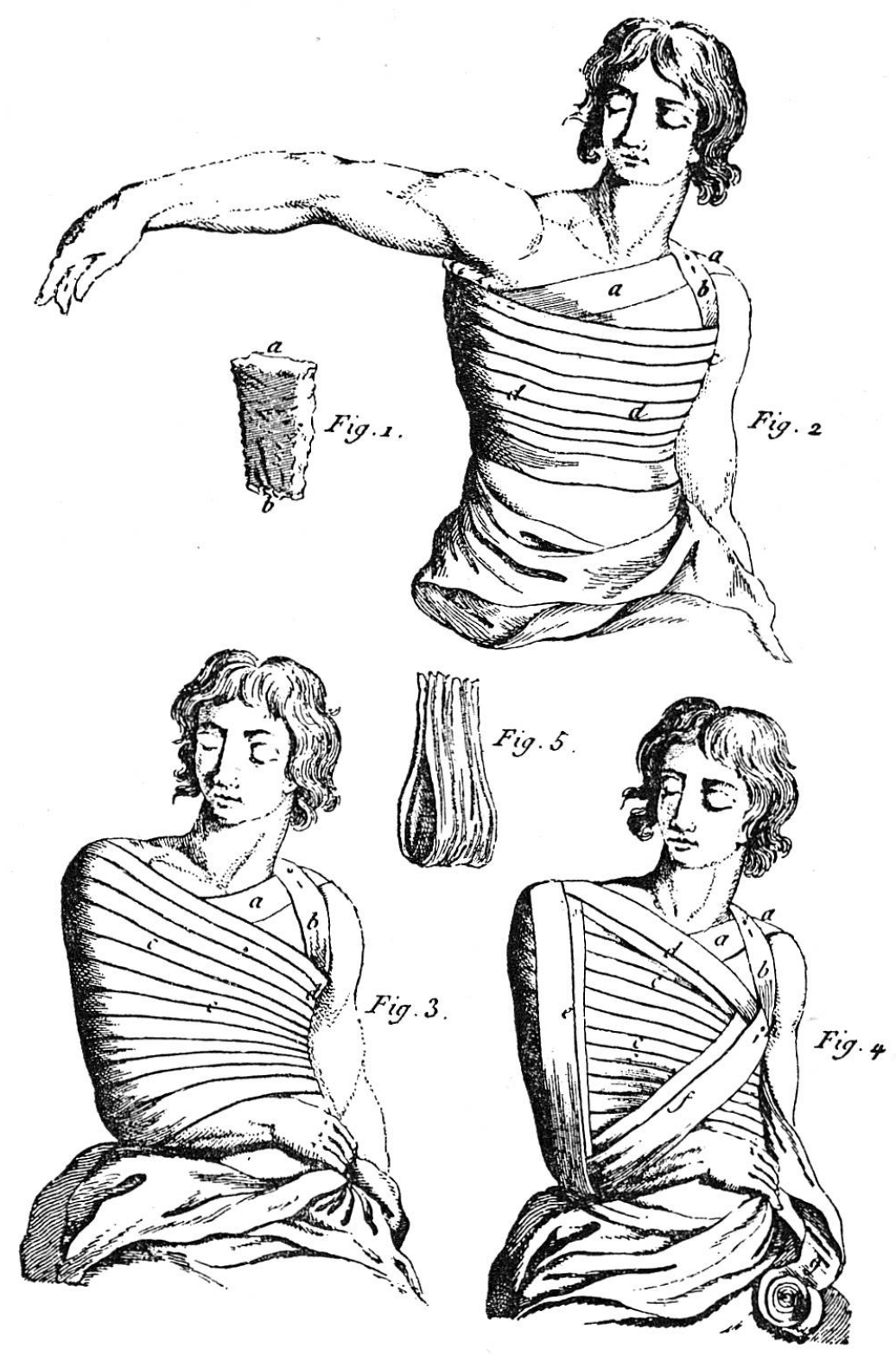

Abb. 1. Die Darstellung des Desaultverbandes in der Edition von Bichat

Der Verband ist mit Fig. 4 noch nicht abgeschlossen. Nach Desaults ursprünglicher Anweisung wurde der Bindenrest der III. Lage des Verbandes zu zirkulären Thorax-OberarmWicklungen verwendet, um ein Abrutschen der Touren e zu verhindern. Als Tragschlinge für die Hand wurde die Schärpe (Fig. 5) benutzt, die an den Touren d befestigt wurde 
Unter diesen Umständen muß die Frage erlaubt sein, ob der «Desault» innerhalb der gültigen Verbandstechnik etwas absolut Neues, eine völlig originale Leistung seines Erfinders darstellt, oder ob nicht auch er nur eine Weiterentwicklung aus den gegebenen Möglichkeiten bedeutet. Denn nicht allein die Verbandsmethoden sind meist alt und stammen zu einem nicht geringen Teil aus der antiken Medizin, auch der Brauch, diese Kunst in Lehrkursen weiterzugeben, wird bis auf die hippokratischen Ärzte zurückgeführt, so man Galens Bemerkung Glauben schenken darf, schon HippoKRATES habe an in Holz nachgebildeten menschlichen Figuren oder an den Körpern von Sklaven Übungen im Bandagieren vornehmen lassen ${ }^{11}$. Selbst die namentliche Bezeichnung zahlreicher Verbände geht auf antike Tradition zurück, und seit Vidus VIDIUS, der Leibarzt des französischen

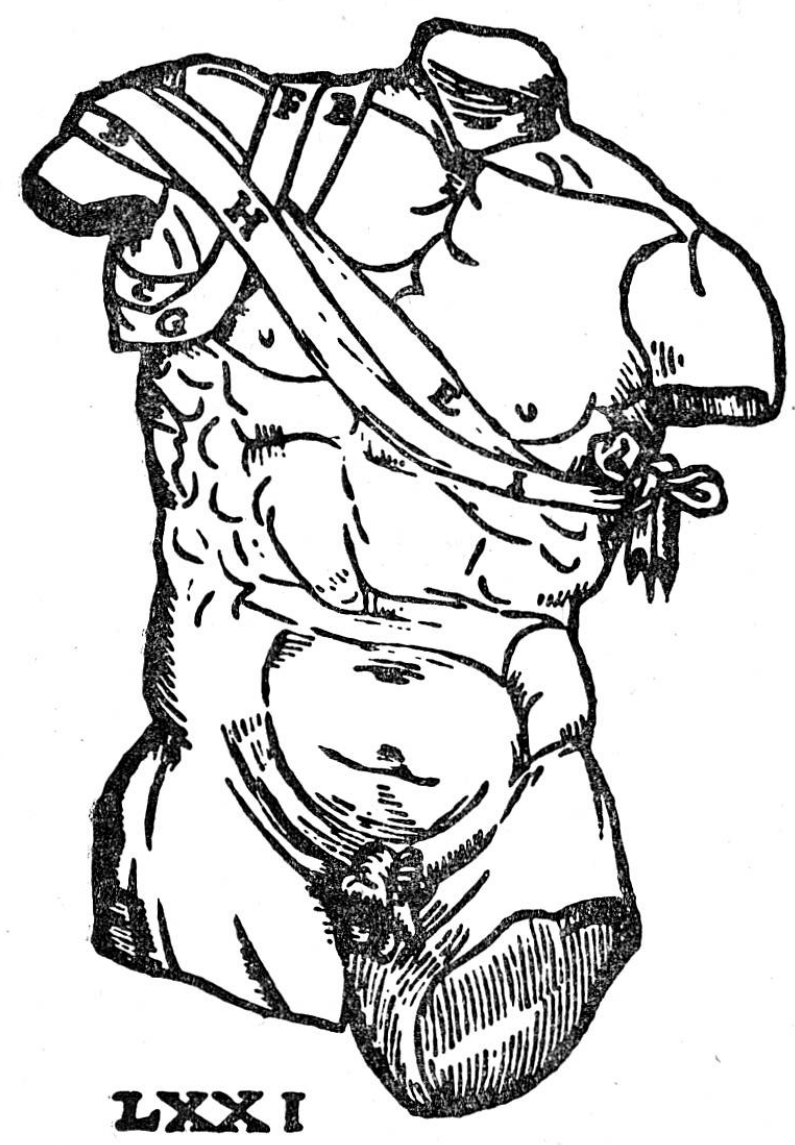

$A b b$. 2. Spica des Glaukias; später nach GALEN benannt

Auch bei ihr wird zuvor ein aus weicher Wolle zusammengerolltes Knäuel in die kranke Achsel gelegt. Der fertige Verband hat aus wesentlich mehr derartigen Spica-Achter-Touren bestanden, wie von dem Zeichner angegeben

11 GALEN in seinem Kommentar zur hippokratischen Schrift De medici officina, Buch I, Abschnitt I (XVIII B 630, ED. KüHN). 
Königs Franz I. und spätere Professor für Philosophie und Medizin an der Universität Pisa, mit seinem berühmten Übersetzungswerk zum Vermittler dieses alten Wissens wurde ${ }^{12}$, standen der europäischen Medizin die griechischen Quellen direkt zur Verfügung. Aus ihnen mag auch mancher Modifikationsversuch des Claviculaverbandes seine Anregung erhalten haben.

Unter den drei überkommenen antiken Verbandslehren, der des Soran, des Heliodor und der unter Galens Namen überlieferten Schrift fordert die letztgenannte mit ihren von der Spica des Glaukias ausgehenden Schulter- und Thoraxverbänden (Abb. 2) zu einem Vergleich mit den einzelnen Lagen des Desaultverbandes heraus ${ }^{13}$.

Betrachtet man den Kataphraktes ${ }^{14}$, den sogenannten Panzerverband mit zwei Bindenköpfen (Abb. 3 a), so sticht seine Ähnlichkeit mit der I. Lage

12 Vidus Vidius, Chirurgia è Graeco in Latinum conversa, Erstausgabe, Paris 1544. Das Werk enthielt die Übersetzung der hippokratischen Schriften Über die Wunden, Über die Fisteln, Über die Kopfwunden sowie Über die Knochenbrüche, Über die Gelenke und Über die ärztliche Werkstätte samt den galenischen Kommentaren, ferner die pseudogalenische Schrift $\ddot{U}$ ber die Verbände und schließlich aus Oribasius Über die Schlingen und Über die Maschinen. Näheres siehe: W. Brockbank, The Man who was Vidius. Annals of the Royal College of Surgeons of England, 19 (1956) 269-95.

${ }^{13}$ Herm Professor K. Schubring habe ich für die freundliche Überlassung des noch unveröffentlichten, nach den Handschriften korrigierten Textes von De fasciis als Grundlàge für die folgenden Abschnitte recht herzlich zu danken. Bei seinen Untersuchungen kam Schubring zu dem Ergebnis, daß die Schrift nicht Galen selbst zugehören kann, was er in seiner bevorstehenden Edition ausführlich begründen wird. - Die Verweisstellen werden der Auffindbarkeit halber weiter nach der Edition von KüHN gegeben, in der allerdings die Namen der Verbände fehlen, während die Ausgabe von Charterius (Hippocratis Coi et Claudii Galeni Pergameni archiatron opera, Paris 1639-1679, XII 470) die Verbandsnamen angibt.

Abb. 2 und die folgenden Abbildungen zu der (pseudo-)galenischen Schrift stammen aus dem zitierten Werk des Vidius. Als ihren Urheber vermutet man den Künstler FraNcesco Primaticcio aus Bologna. Der Meister, der nach Primaticcios Zeichnungen die Holzschnitte anfertigte, ist wahrscheinlich FrançoIs Jollat gewesen (vgl. W. BrocKBANK, a.a.O., S. 277). Zum Verständnis der Abbildungen sei erwähnt, daß sie seitenverkehrt von den Druckstöcken abgezogen sind, was an den doppelseitigen Verbänden, z.B. Abb. 3, ins Gewicht fällt. Das läßt sich nicht nur aus der Reihenfolge der einzelnen Bindentouren erschließen, sondern wird auch durch die Originalzeichnungen bewiesen, die in der Pariser Handschrift erhalten sind (Hs. 6866; vgl. H. OмоNт, Collection de chirurgiens grecs avec dessins attribués au Primatice, Paris o. J.).

Die Skizzen des Desaultverbandes sind dem Atlas und Grundriß der Verbandlehre von A. Hoffa und R. Grashey (München 1914, Tafel 55-7) entnommen.

${ }^{14}$ De fasciis c. 86 (XVIII A 817). 


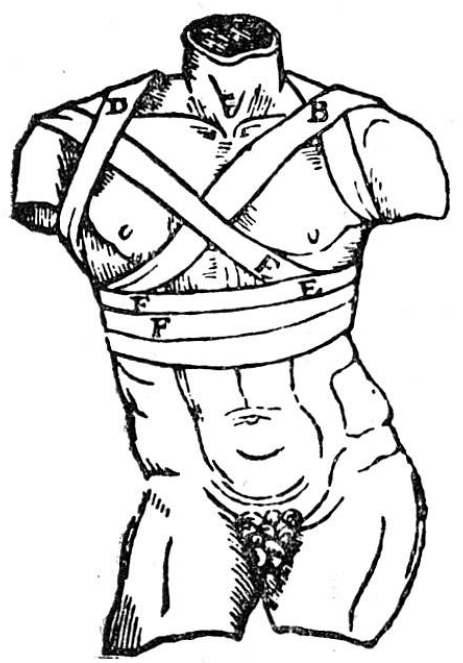

IXXX:

a

Der Kataphraktes mit zwei Bindenköpfen nach der Beschreibung der (pseudo-)galenischen Schrift in der Abbildung bei Vidius. Der Verband gewinnt seinen Halt durch Spicatouren über beiden Schultern

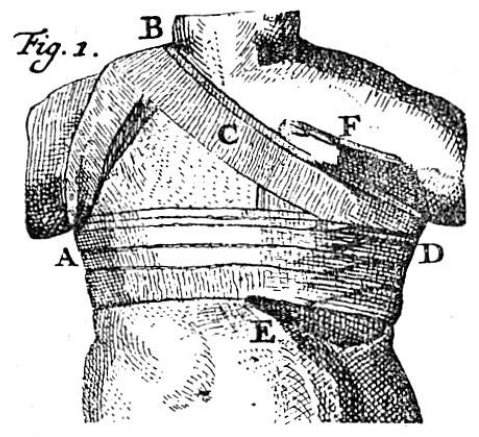

b Der Kataphraktes bei Heister als Brustverband mit einer Spicatour lediglich über der Schulter der gesunden Seite

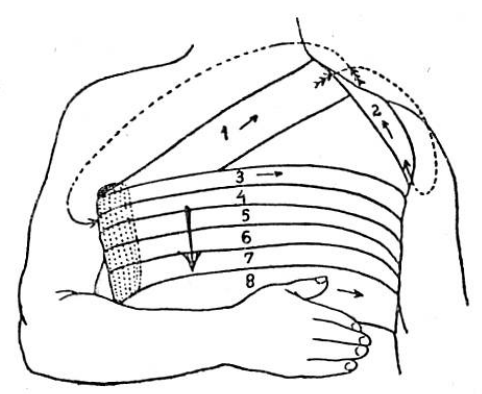

c

Die erste Lage des Desaultverbandes nach der Verbandslehre von HoFraGrashey (Skizze zu Tafel 55)

$A b b$. 3. Die erste Lage des Desaultverbandes und ihre Vorgänger

des «Desault» ins Auge (Abb. 3c). Von ihm heißt es in der pseudogalenischen Schrift, die Mitte der zu zwei Köpfen aufgerollten Binde wird unter der Achsel angelegt, und die Bindenköpfe müssen sich halsnah auf der gleichseitigen Clavicula x-förmig kreuzen. Alsdann wird der eine Bindenkopf am Nackenansatz über den Rücken zur gegenüberliegenden Schulter und unter der Achsel durchgeführt, so daß auf der anderen Clavicula gleichfalls ein X entsteht. Der Rest der Bindenköpfe wird in Kreistouren um den Thorax aufgewickelt. Ein Blick auf beide Abbildungen genügt, um zu erkennen, daß hier von Desault lediglich die Spicatour um die kranke Schulter weggelassen wurde. Aber auch dies ist nicht einmal sein eigener Gedanke; 
denn bereits Heisten gibt den Kataphraktes mit lediglich einer Spicatour über die Schulter der gesunden Thoraxhälfte bei Mammaoperationen an $(\mathrm{Abb} .3 \mathrm{~b})^{15}$. Originell ist also lediglich Desaults Idee, auch bei einer Claviculafraktur die Schulter der verletzten Thoraxseite von einer Spicatour zu entlasten. Der Zweck, den er mit dieser ersten Verbandslage verfolgte, ist bereits bei WARDENBURG ausführlich erklärt ${ }^{16}$ : Sie soll den Brustkorb möglichst ruhigstellen und die Atemexkursionen auf das Zwerchfell ableiten, damit der Thorax gewissermaßen als Schiene bei der Fixation des Armes durch die II. Lage des Verbandes dienen kann, die in deszendierenden Kreistouren den Oberarm am Rumpf befestigt und zugleich durch Anziehen des unteren Humerusendes gegen den Brustkorb einen Zug des peripheren Claviculafragments nach außen bewirken soll (durch Hebelwirkung über das in die Achsel eingelegte Keilkissen als Hypomochlion) (Abb.4a). Grundsätzlich führte Desault also mit den beiden unteren Lagen seines Verbandes die Ruhigstellung durch: Mit der I. Lage die Ruhigstellung des Thorax in seiner Eigenbewegung, mit der II. aber die Fixation des Oberarmes am ruhiggestellten Brustkorb. Sein Ziel, durch einen Reduktionszug nach hinten, nach außen und nach oben die Repositionsstellung zu erhal-

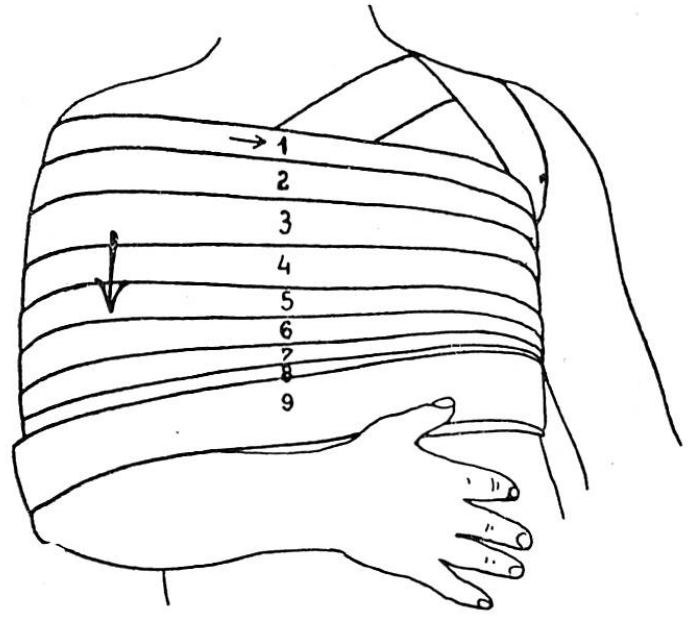

a

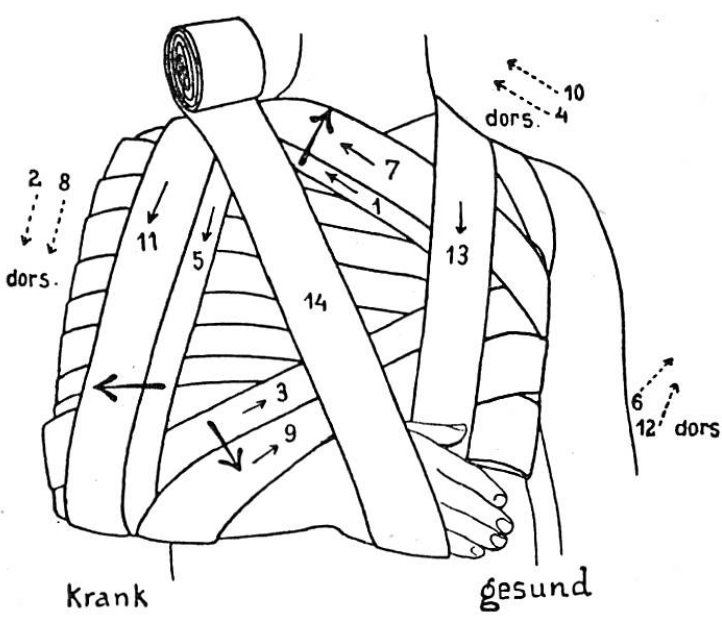

b

$A b b .4$. Die zweite Lage und die dritte Lage des Desaultverbandes

15 D. L. Heister, a. a. O. Tab. XXXVIII S. 1178 (Erklärung S. 1176).

16 WARDENBURG, a.a. O., S. 124. 
ten ${ }^{17}$, verwirklicht die II. Lage des Verbandes nur zum Teil. Mit ihr sollte lediglich der bereits charakterisierte Zug nach außen und bei entsprechender Haltung der Schulter durch einen Gehilfen ein gewisser Zug nach hinten erreicht werden. Erst die III. Lage erfüllt die restlichen Forderungen (Abb. 4b, Pfeile).

Bereits die hippokratische Verbandstechnik kannte die Unterteilung in eine Hypodesis und eine Epidesis, einen Unter- und einen Oberverband. In der Schrift Von der ärztlichen Werkstätte heißt es von der Hypodesis, sie habe das Ziel, «voneinander Wegstehendes zueinander zu bringen oder Auseinandergewichenes zusammenzuführen, auch Zusammengestauchtes auseinanderzuziehen oder Verrenktes einzurichten ${ }^{18}$. Der Anfang des Oberverbandes - der Epidesis oder des Epidesmos - hatte an einer sicheren Halt gewährenden Stelle angelegt zu werden und sollte den Gesamtverband befestigen ${ }^{19}$. Auch die Hippokratiker kombinierten also bereits Teilverbände miteinander zu einem Gesamtverband. Aber umgekehrt wie bei Desault hatte der Unterverband das Repositionsergebnis und der Oberverband die Ruhigstellung zu garantieren. Dies galt jedoch primär nicht etwa für die komplizierteren Bandagierungen, sondern grundsätzlich und ursprünglich für die einfachen Frakturverbände der Gliedmaßen, an denen die Reihenfolge: Repositionsverband - Fixationsverband entwickelt worden war. Hier dürfte sie in einer Traumatologie ohne Gipstechnik auch zweckentsprechend gewirkt haben, und so fußt noch Ambroise Paré in seiner Verbandslehre auf den hippokratischen Anweisungen ${ }^{20}$. Von den Extremitäten aber muß bei den Alten diese Verbandsanordnung unverändert auf die mechanisch völlig anderen Verhältnisse am Rumpf übertragen worden sein, und in diesen schwer ruhigzustellenden Körperregionen wurde der fixierende Oberverband zugleich zum Abstützungsmittel: Er sollte etwa an Brust, Rippen und Kopf oder an ähnlich beschaffenen Körperabschnitten Erschütterungen vermeiden, teils von seiten des Pulses, ferner auch bei Husten, Niesen oder einer anderen Bewegung, wie solche an Brust und Schädel zu geschehen pflegen ${ }^{21}$. Es dürfte ohne weitere Erklärungen einleuchten, daß hier eine solche Reihenfolge der Teilverbände ihren Sinn verlor, aber dennoch wurde

17 Vgl. Bichat, Euvres chirurgicales de P.J. Desault, S. 71.

18 De medici officina c. 11 (Littré III, 306).

${ }^{19}$ Ebenda c. 9 (Littré III, 298f.).

20 Ambrosius Paraeus, Opera Chirurgica. Frankfurt 1594, 1. XIII, c. 3, p. 397.

${ }^{21}$ De med. officina c. 25 (Littré III 334). Vgl. auch GaLens Kommentar Buch III, Abschnitt XXXVII (XVIII B 917 ff., ED. KüHN). 
sie als genereller Grundsatz der Verbandstechnik über das gesamte Altertum beibehalten.

Betrachtet man unter diesen Voraussetzungen die (pseudo-)galenische Verbandslehre, dann stellte die Spica des Glaukias (Abb.2) den Unterverband dar. Sie besaß also die Aufgabe, die Claviculafraktur oder Schulterluxation - denn bei beiden Verletzungen wurde sie angewandt - in Repositionsstellung zu halten, dürfte aber beim Schlüsselbeinbruch kaum ihren Zweck erfüllt haben. Als Epidesis dienten die Variationen des Armschleuderverbandes mit zwei Bindenköpfen (Abb. $5 \mathrm{a}, \mathrm{b}, \mathrm{c})^{22}$. Ihnen wurde also die Halte- und Sicherungsfunktion für Thorax und Oberarm zugeschrieben. Den einzelnen Variationen kommt sicherlich keine eigene Bedeutung zu,

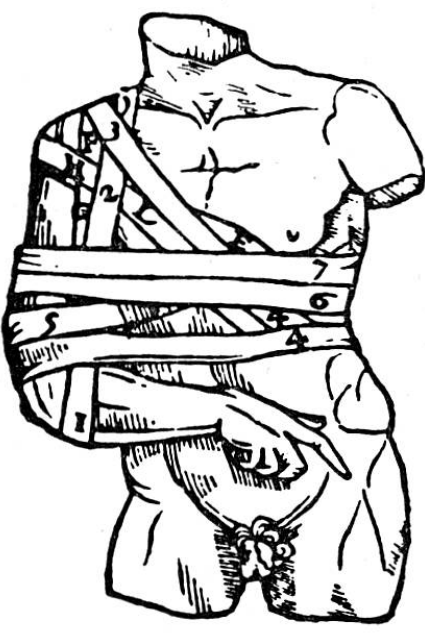

LXXII

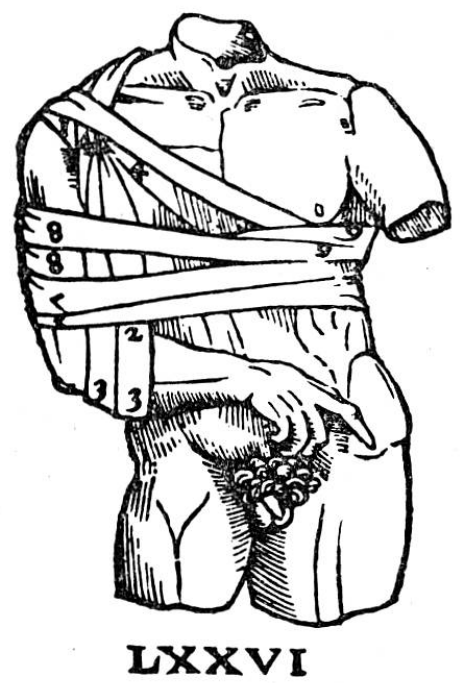

b

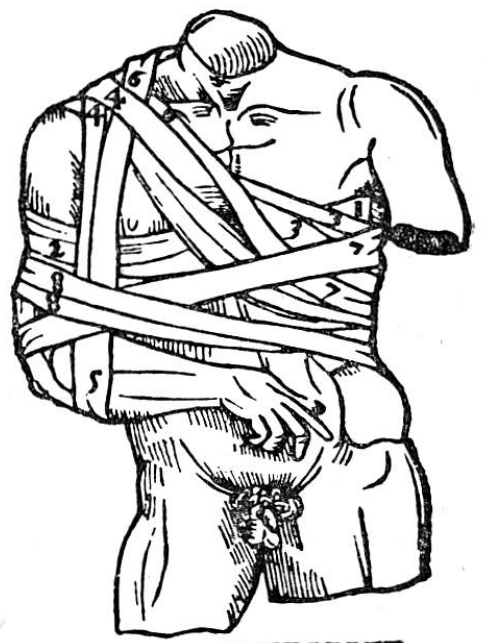

LXXVII

$\mathbf{a}$

Abb. 5. Die «armhaltende Schleuder» mit zwei Bindenköpfen in ihren drei Variationen

Sie bildete den Oberverband über der einfachen Spica des Glaukias (Abb. 2). Die zirkulären Touren um Brustkorb und Oberarm sind wiederum unvollständig gezeichnet. Für sie wird in der (pseudo-)galenischen Verbandslehre ausdrücklich die Anweisung gegeben, sie müßten den gesamten Oberarm bedecken. Die arabischen Ziffern bezeichnen die Touren des Schleuderverbandes, die lateinischen Buchstaben in 5 a die Touren der darunter angewickelten Spica

${ }^{22}$ Die Verbandsanweisungen siehe GaLen, De fasciis c. 78, 82 und 83 (XVIII A 814 und 815 ED. KÜHN). Schubring ordnet c. 78, auch verbandstechnisch sinnvoller, vor c. 82 und 83 ein. Die Zeichnungen bei Vidius entsprechen nicht in allem genau den Anweisungen der Verbandslehre. 
sondern $5 \mathrm{a}-\mathrm{c}$ bauen verbandstechnisch aufeinander auf, wobei sich die Zahl der Bindentouren von einer Variation zur anderen jeweils vermehrt. Außerdem unterscheiden sie sich durch den Anfang der Wickelung; denn bei 5 a wird die Mitte der auf zwei Köpfe aufgerollten Binde unter dem angewinkelten Unterarm angelegt, die Bindenköpfe werden vorn und hinten zur kranken Schulter und von dort unter $\mathrm{x}$-förmiger Kreuzung über der Clavicula brust- und rückenwärts hinten unter die gesunde Achsel gewickelt, von wo sie in Kreistouren um Thorax und Oberarm ausmünden, während $5 \mathrm{~b}$ mit der Bindenmitte auf der kranken Schulter beginnt und die Bindenköpfe abwärts zum Unterarm und wieder aufwärts zur kranken Schulter führt, von wo sie wie bei 5 a weiterlaufen. 5 c schließlich beginnt mit der Bindenmitte unter der gesunden Achsel, macht mit beiden Bindenköpfen eine Kreistour um Rippen und Oberarm zurück zur gesunden Achsel und von dort eine Schrägtour aufwärts zur kranken Schulter, von wo beide Bindenköpfe wie bei $5 \mathrm{~b}$ in gerader Richtung abwärts zum Unterarm geführt werden und weiterlaufen wie bei $5 \mathrm{~b}$ und $5 \mathrm{a}$.

Sieht man nunmehr von dem Zweck ab, den die III. Lage des «Desault» und der Schleuderverband der Alten jeweils erfüllen sollten, so läßt sich in verbandstechnischer Hinsicht die Ähnlichkeit in der Bindenführung zwischen $5 \mathrm{c}$ und III. Desaultlage nicht verkennen. Aus der doppelköpfigen ist allerdings eine einköpfige Binde geworden. Aber auch sie beginnt in der gesunden Achsel, verzichtet verständlicherweise auf die Kreistour um Oberarm und Brustkorb, da diese Aufgabe von der II. Lage des Verbandes übernommen wurde, steigt somit unmittelbar vorn zur kranken Schulter hinauf und hinten längs des Oberarms zum Unterarm hinab. Hier ist nun eine neue Bindentour vom humerusnahen Unterarmende direkt zur gesunden Achsel zwischengeschaltet, einmal um den Zug des peripheren Claviculafragments nach außen noch zu verstärken, zum anderen aber, um die Touren nachzuholen, die mit zwei Bindenköpfen gleichzeitig gewickelt werden können. Die Binde führt daher jetzt von der gesunden Achsel am Rücken entlang zur kranken Schulter, dann vorn, längs des Humerus, zum Unterarm und schräg am Rücken entlang zur gesunden Achsel zurück. Diese Ähnlichkeit, bei der sich die III. Lage des «Desault» deutlich als eine schöpferische Fortentwicklung des alten Armschleuderverbandes zu erkennen gibt, dürfte noch stärker bei einem Vergleich mit den alten Bildtafeln Desaults zum Ausdruck kommen (Abb. 1, Fig. 4), wenn man bedenkt, daß hier das Bindenende noch nicht zu einer Tragschlinge für die Hand benutzt, sondern in Kreistouren um Oberarm und Thorax aufgewickelt wurde. 
Fassen wir die Einzelheiten dieser Untersuchung zusammen, so läßt sich folgendes feststellen: Die I. Lage des Verbandes entstand dadurch, daß dem Kataphraktes der antiken Verbandslehre die Spicatour über der verletzten Schulter genommen wurde. Die II. Lage wurde aus der ersten Kreistour der alten Armschleuder um Oberarm und Brustkorb zu einem eigenen Teilverband entwickelt, der Humerus und Thorax in ihrer ganzen Ausdehnung umfaßte. Die III. Lage entstammte einer Modifikation des zweiköpfigen Armschleuderverbandes in eine einköpfige Wickelung, bei der freilich der neue Weg vom Unterarm zur gesunden Achsel brust- und rückenwärts eine entscheidende Rolle spielen dürfte. Desault bewegte sich also völlig in den Bahnen der überkommenen Verbandstechnik und schuf dennoch für die Behandlung des verletzten Schultergürtels grundsätzlich neue Bedingungen: Ruhigstellung des Thorax und Fixation des Oberarmes verlegte er nicht allein in die unteren Schichten des Verbandes, er trennte auch beide Funktionen, die im Altertum stets als einheitlicher Verbandsakt aufgefaßt wurden, und versuchte diesen Aufgaben mit zwei verschiedenen Lagen des Verbandes gerecht zu werden. Auch befreite er die Claviculafraktur endgültig von dem schädlichen Druck der Spica nach innen unten. Aus der alten Armschleuder schließlich wurde ein sicherer Verband des Schulter-Oberarm-Bereiches in Korrekturstellung, und wenn sich auch für den Schlüsselbeinbruch mit ihm nicht alle Hoffnungen auf eine geeignete und exakte Ruhigstellung erfüllten, so ging der «Desault» doch in das Repertoire unserer großen Verbände ein und wurde selbst in die Gipstechnik übernommen.

So dürfte denn auch das Ergebnis dieser Untersuchung die Originalität des Verbandes kaum in Zweifel ziehen, und sie verfolgte auch keineswegs das Ziel, die alten Prioritätsstreitigkeiten wieder aufleben zu lassen, mit denen sein Schöpfer hier und da zu kämpfen hatte ${ }^{23}$. Aber sie zeigt zugleich

${ }^{23}$ BrünNinghausen in seiner Abhandlung über den Schlüsselbeinbruch (Würzburg 1791, zit. nach Wardenburg, a.a.O. S. 122 und 126) wollte Paré die Priorität zuerkennen. Verständlicherweise konnte in dieser Untersuchung nicht den zahlreichen, mehr oder weniger ephemeren Modifikationen der Spica nachgegangen werden. Ein Blick auf den entsprechenden Abschnitt bei PARÉ (an bereits zitierter Stelle 1. XIV c. 8 p. 407) zeigt die Haltlosigkeit dieser Behauptung. Paré muß wie bereits mancher vor ihm wohl den schädlichen Druck der Spica erkannt haben, verbindet selber aber wiederum mit einer doppelköpfigen Binde, die sich über der verletzten Clavicula x-förmig (decussatim) kreuzt. Allerdings versucht er dann mit der Binde einen $\mathrm{Zug}$ nach hinten-außen auszuüben und befiehlt dem Patienten, die Hand der verletzten Schulterseite hinten auf die Hüfte zu legen und so den Schulterarmbereich nach hinten zu biegen. 
doch deutlich, daß auch dieser Verband nur eine Fortentwicklung, ein Glied in der Kette einer Tradition darstellt, die bis in die Antike zurückreicht, und GrüNDER hatte nicht einmal Unrecht, wenn er bereits vor hundert Jahren in seiner Geschichte der Chirurgie die Vermutung äußerte, die Spica GaLens für den Schlüsselbeinbruch sei vielleicht auch Ursache zur Erfindung dieses Verbandes gewesen ${ }^{24}$. Selbst Desault scheint sein Werk in keinem anderen Licht gesehen zu haben; jedenfalls schreibt Bıснат : «Desault chercha pour cet appareil, comme pour ses autres bandages, à multiplier l'application des moyens connus, sans en créer de nouveaux ${ }^{25}$.

${ }^{24}$ J. W. L. GrüNDER, Geschichte der Chirurgie von den Urzeiten bis zu Anfang des achtzehnten Jahrhunderts. Breslau 1865, S. 72.

${ }^{25}$ Bichat, a. a. O., S. 71. 